\title{
The Consumers Characteristics Analysis of Low Temperature Home Delivery
}

\begin{abstract}
Shu-Fang Lai ${ }^{1}$
Abstract: Because of technological advancements and the popularity of the Internet, online shopping has become an important shopping channel for consumers. Because people increasingly eat out, more consumers shop online, and food products are collected from convenience stores, or frozen food home delivery services are used. This study used questionnaire surveys to analyze the consumption habits of residents who shop online for frozen foods in the urban areas of northern Taiwan (Taipei City and New Taipei City). We distributed and collected 548 questionnaires, of which 484 were valid. Descriptive statistics, a chi-square test, and logistics regression analysis were used to analyze consumer characteristics, as well as important influential factors. The research results indicated that most online shoppers were women, and the top 3 factors influencing their purchasing decisions were freshness, delivery convenience, and ordering convenience. Participants in the age group of 40-49 years old, living in the urban area of the New Taipei City, without junior college education, and with less than 10,000 NTD monthly incomes, were less likely to purchase frozen foods using low-temperature logistics services.
\end{abstract}

Keywords: Consumer characteristics, frozen food, online shopping, home delivery, logistics.

\section{Introduction}

As the average income rises in Taiwan, and Internet use become more ubiquitous, the entire social environment and structure is changing, leading to changes in contemporary lifestyles. Advances in technology and globalization have driven consumption rates to higher levels, and as a result people are gradually eating out more. The Otaku economy (stay-at-home-economy) is on the rise. People often find online shopping convenient, because they can receive the goods without leaving home, or they can collect their food purchases from convenience stores in the area. According to data from the Department of Statistics in the Ministry of Economic Affairs [4] between 1997 and 2004 the sales volume of frozen food in Taiwan declined year on year, and only increased again from 2004. By 2011, the production value was approaching 52.8 billion NTD (including non-frozen processed foods), and consumers tended to purchase frozen meat and processed foods. Because of the rise of online shopping, and the rise of the new home-delivery logistics industry, the Taiwanese public is increasingly opting for frozen foods. Online shopping is usually linked with home delivery services that deliver purchases directly to the customer. According to Kuo and Chung [7], home delivery of frozen foods made up $10 \%-15 \%$ of all home food deliveries at the time, and was expected to reach $30-50 \%$ with a market value of more than 6 billion NTD in 2010.

1. Department of Logistics Management, Takming University of Science and Technology, No.56, Sec.1, Huanshan Rd., Neihu District, Taipei City, Taiwan 11451, R.O.C.

Email: sflai@takming.edu.tw, cain1003@gmail.com
This demonstrates the importance of frozen food home deliveries as part of the entire home delivery industry. This study therefore evaluates the lowtemperature home delivery industry and its related logistics; to analysis the factors influencing consumers' purchase decision-making, frozen food consumer characteristics, as well as the scale of the home delivery market. The objective of this study is to provide low-temperature logistics companies and online retailers with data on consumer characteristics and demands in this market, to be used as a reference in planning and adjusting operational strategies.

This study evaluated the literature on low-temperature foods, home delivery, and online shopping. Wang [14] explored the delivery routes of lowtemperature foods, and demonstrated that because frozen food involves temperature control, mathematical models can be used to address this problem, using time-window restrictions to represent the preservation and sales characteristics of frozen food, as well as generic algorithms to achieve the minimum total transportation costs and maximum customer satisfaction. Guo [5] examined consumer characteristics and online shopping intentionoffor fresh foods in a study on white-collar workers in three metropolitan areas (Taipei, Taichung, and Kaohsiung), and their willingness to purchase fresh food online. The results demonstrated that consumers in Taipei were more willing to buy fresh foods online, and that significant differences existed among areas. Chen [3] studied the frozen processed food industry to explore the relationship between performance and four variables: raw material selection; production and manufacture; delivery; and marketing of frozen food. 
Forty-two Taiwanese frozen processed food businesses were used for the analysis. Chang's [2] study evaluated the effect of new low-temperature foods on market performance by focusing on the acceptance of new products in the market, and cwithducted indepth interviews in eight companies. The research results indicated that the introduction of new products was determined by customer-oriented approach, and corporations need to keep promoting new products to maintain the performance of old and new products on the market. Yeh [15] researched the behavior, satisfaction, and loyalty of frozen goods consumers and found that the main customers of frozen foods were in the 20-35 age group, married with children, and had university/college level education, worked as private employees, and had a personal monthly income of 30,000 to 40,000 NTD.

Literature focused on home delivery services includes Lu's [11] analysis of conditions in the Taiwanese home delivery market. That study integrated research opinions in the industry, government, and academia, to establish home delivery service management strategies for home delivery operators to use as a reference in their operational strategies. The research results demonstrated that the three most important management principles were to construct comprehensive delivery network energy, elevate service quality, and strengthen service mechanisms. Strategic plans have to prioritize customer development, but other strategies for service optimization were also found to be essential and need to be integrated to achieve an optimal strategic plan. Lee [9] and Lan [8] investigated service quality and satisfaction in the home delivery business by analyzing productivity and marketing strategies. The study aimed to provide businesses with a basis to adjust marketing strategies with objective and quantitative results when conducting productivity performance indicator analyses. Yu [16] explored the key factors influencing management success in the home delivery industry, using Taiwan Pelican Express as a case study, and conducted in-depth interviews and questionnaire surveys to gather data. The study determined the key factors influencing management success in the home delivery industry by using Aaker's [1] findings, which demonstrated that governments need to implement relevant regulations to enable the construction of comprehensive delivery networks, elevate service quality, strengthen employee training, apply advanced technology, establish comprehensive customer complaint mechanisms, product innovation, and diverse services, and evaluate whether these factors were also keys to success for home delivery businesses in Taiwan. Hu and $\mathrm{Wu}[6]$ analyzed the effect of the brand images of home delivery services on consumer purchase intentions. A questionnaire survey based mainly on three prominent home delivery companies in Taiwan was conducted, and used as a structural equation model to analyze them. The findings indicated that consumer brand image and brand alliance compatibility affect purchase intention, and when customers select home delivery companies, a high degree of brand alliance compatibility can elevate the image of a company's brand.

Shiaw and Chiang [12] explored possible factors affecting online shopping behavior, by interviewing internet users in southern Taiwan, and found that the characteristics that companies exhibited online were the most influential and significant, and product characteristics were more specific, especially online transaction security and procedural convenience provided by online companies. Lin [10] analyzed the effect of convenience stores (both virtual and physical channels) on consumption behavior from the viewpoint of consumers. The results demonstrated that online retail options and the collection of produce at convenience stores have gradually become approved and accepted by consumers. Therefore, if businesses could focus more on transaction security, greater business opportunities could follow. In addition, the results showed that promotional activities can encourage consumers to make use of convenience stores' collection services, and people preferred cash discounts and free logistics services as promotional items.

\section{Methods}

\section{Research Methods and Questionnaire Design}

To analyze the characteristics of online consumers of low-temperature products, this study used questionnaire surveys as a data collection method. In addition, descriptive statistics, the chi-square test, and logistics regression were used in the analyses. The sampling method was quota sampling in nonprobability sampling methods, and sample numbers were therefore planned and based on the populations of age groups in different areas. Furthermore, convenience sampling was used for the surveys.

\section{Questionnaire Design}

To analyze the consumer characteristics, this study established six basic hypotheses, and the questionnaire questions were designed based on the data item needed for each basic hypothesis.

$\mathrm{H}_{1}$ : Subjects with different characteristics differ in purchase frequency in their use of low-temperature logistics to buy frozen food.

$\mathrm{H}_{2}$ : Subjects with different characteristics differ in mean purchase amount in their use of lowtemperature logistics to buy frozen food. 
$\mathrm{H}_{3}$ : Subjects with different characteristics differ in the purchase experience in their use of lowtemperature logistics to buy frozen food.

$\mathrm{H}_{4}$ : Subjects with different characteristics differ in purchase channels in their use of low-temperature logistics to buy frozen food.

$\mathrm{H}_{5}$ : Subjects with different characteristics differ in delivery methods in their use of low-temperature logistics to buy frozen food.

$\mathrm{H}_{6}$ : Subjects with different characteristics differ in payment methods in their use of low-temperature logistics to buy frozen food.

Based on these six hypotheses, the questionnaire was divided into three portions. The first section focused on frozen food purchases, the second, on experiences using low-temperature logistics services, and the third included basic data. To ensure that the questionnaire content was suitable and easy to understand, it's not only used expert validity evaluation, but also trials to ensure that the question content was clearly explained and easy to understand. After two trials and content adjustments, the questionnaire content was verified for the official survey.

Consumers in metropolitan Taipei have significantly greater purchase intention than consumers in other metropolitan areas. Taipei City and New Taipei City were therefore selected as the areas to be surveyed. We distributed 300 questionnaires, and ensured that the sample size conformed to the population. The demographic ratios of different genders and ages at the end of 2011 in Taipei County was therefore used for non-probabilistic quota sampling to compute the number of samples that had to be extracted from each age group, and the groups with fewer than 30 questionnaires were adjusted to 30 questionnaires. Table 1 demonstrates that this study distributed 382 questionnaires, approximately 30-37 questionnaires for each age level.

\section{Results and Discussion}

In December 2012, surveyors performed visits to conduct the questionnaire survey, and retrieved a total of 548 questionnaires, with 484 valid questionnaires, constituting a valid retrieval rate of $88.32 \%$. The age group distribution of the valid questionnaires conformed to the planned distribution ratio, as indicated in Figures 1 and 2.

\section{Descriptive Statistics}

\section{Basic Data}

Approximately half of the participants were from New Taipei City, and the other half from Taipei City
(57\% and 43\%). In terms of gender, the sample group had slightly more women (54\%). More participants were married (57\%) than unmarried. Fifty-five percent had children, and 38\% had high school or vocational school as their education level. Thirty-eight percent of the participants were working in commerce/services. Forty-one percent had a mean monthly income in the 20,001-40,000 NTD bracket. Most had Internet experience, and time spent online each week was less than 3.5 hours. Forty-seven percent of participants had online shopping experience (Table 2 ).

\section{Frozen Food Purchase}

The analytical results demonstrated that 135 participants had used low-temperature logistics services to purchase frozen food (28\%), and 349 had never used them. Among the non-users, the main reasons for not using low-temperature logistics services to purchase frozen food included the inability to see the physical product, quality concerns, and concerns about food storage and hygiene.

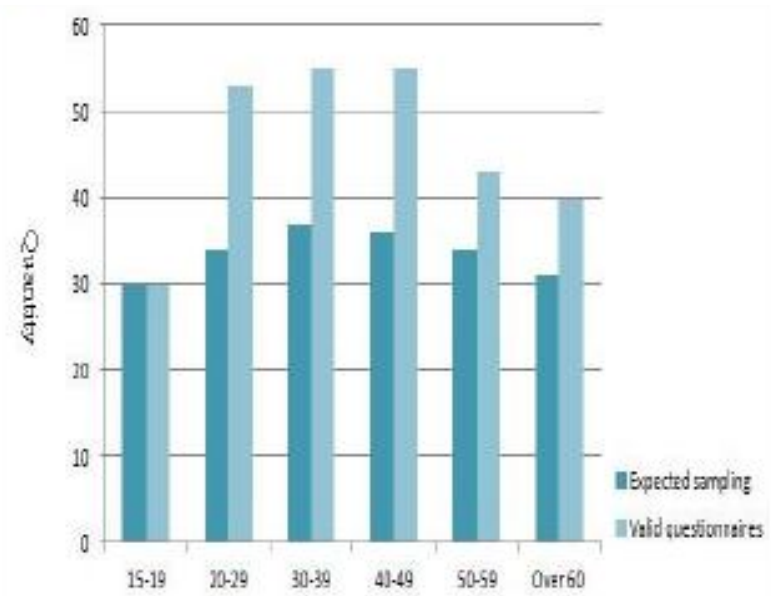

Figure 1. Comparison of New Taipei City questionnaires

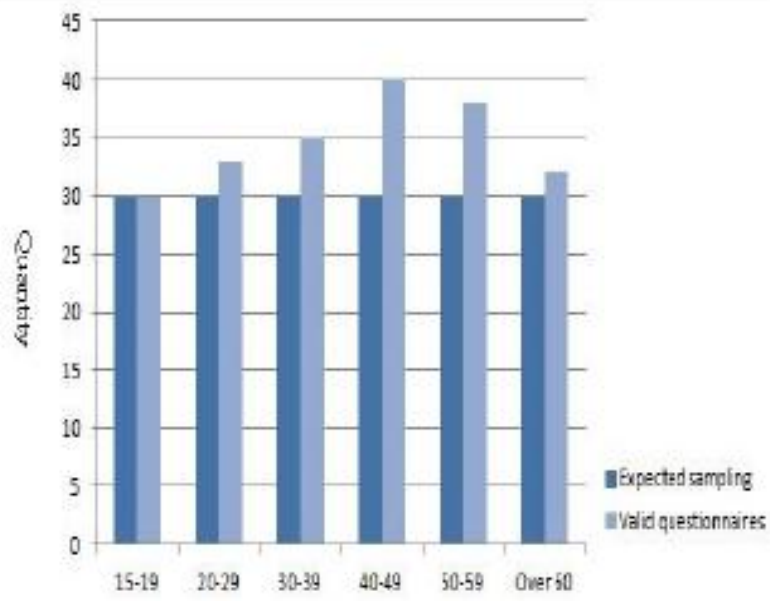

Figure 2. Comparison of Taipei City Questionnaires 
Table 1. Plan for sample questionnaire numbers

\begin{tabular}{llll|lll}
\hline \multirow{2}{*}{ Age } & New Taipei City & & & & \\
\cline { 2 - 7 } & Numbers (note) & Ratio (\%) & Projected & Numbers (note) & Ratio (\%) & Projected \\
\hline $15-19$ & 269,741 & 0.05 & 30 & 159,484 & 0.03 & 30 \\
$20-29$ & 595,867 & 0.11 & 34 & 331,131 & 0.06 & 30 \\
$30-39$ & 695,055 & 0.12 & 37 & 427,287 & 0.07 & 30 \\
$40-49$ & 649,819 & 0.12 & 36 & 432,506 & 0.08 & 30 \\
$50-59$ & 606,550 & 0.11 & 34 & 419,719 & 0.08 & 30 \\
Over 60 & 532,076 & 0.09 & 31 & 497,875 & 0.08 & 30 \\
\hline Total & $3,349,108$ & 0.60 & 202 & $2,268,002$ & 0.40 & 180
\end{tabular}

Note : The numbers of people in different age groups are based on Directorate General of Budget, Accounting and Statistics, Executive Yuan 201

Source: Directorate General of Budget, Accounting and Statistics, Executive Yuan

Table 2. Basic data of subjects

\begin{tabular}{|c|c|c|c|c|c|c|c|}
\hline $\begin{array}{l}\text { Demographic } \\
\text { variable }\end{array}$ & Type & Frequency & Ratio (\%) & $\begin{array}{l}\text { Demographic } \\
\text { variable }\end{array}$ & Туре & Frequency & Ratio (\%) \\
\hline \multirow{2}{*}{ Gender } & Male & 224 & 46 & \multirow{5}{*}{ Education } & \multirow{5}{*}{$\begin{array}{l}\text { Elementary school or less } \\
\text { Junior high school } \\
\text { High (vocational) school } \\
\text { Junior college and } \\
\text { university } \\
\text { Graduate school and over }\end{array}$} & 50 & 10 \\
\hline & Female & 260 & 54 & & & 48 & 10 \\
\hline \multirow{6}{*}{ Age } & 15-19 years & 60 & 12 & & & 185 & 38 \\
\hline & $20-29$ years & 158 & 33 & & & 183 & 38 \\
\hline & \multirow{4}{*}{$\begin{array}{l}30-39 \text { years } \\
40-49 \text { years } \\
50-59 \text { years } \\
60 \text { years and } \\
\text { over }\end{array}$} & \multirow{3}{*}{$\begin{array}{c}148 \\
86 \\
20\end{array}$} & \multirow{2}{*}{$\begin{array}{l}31 \\
18\end{array}$} & & & 18 & 4 \\
\hline & & & & & Industry/manufacturing & 59 & 12 \\
\hline & & & 4 & & Commerce/service & 184 & 38 \\
\hline & & 12 & 2 & & $\begin{array}{l}\text { Agriculture, forestry, } \\
\text { fishery, livestock }\end{array}$ & 5 & 1 \\
\hline \multirow{3}{*}{$\begin{array}{l}\text { Place of } \\
\text { residence }\end{array}$} & Taipei city & 208 & 43 & \multirow{7}{*}{ Occupation } & Self-employed & 32 & 7 \\
\hline & $\begin{array}{l}\text { Taipei County } \\
\text { metropolitan } \\
\text { area }\end{array}$ & 255 & 53 & & $\begin{array}{l}\text { Military, public servant, } \\
\text { education }\end{array}$ & 20 & 4 \\
\hline & $\begin{array}{l}\text { Taipei County } \\
\text { suburban area }\end{array}$ & 21 & 4 & & Home-maker & 53 & 11 \\
\hline \multirow{7}{*}{$\begin{array}{l}\text { Time spent } \\
\text { online each } \\
\text { week }\end{array}$} & $\begin{array}{l}\text { Does not use } \\
\text { the internet }\end{array}$ & 131 & 27 & & Retired & 35 & 7 \\
\hline & $\begin{array}{l}\text { Under } 3.5 \\
\text { hours }\end{array}$ & 103 & 21 & & Student & 70 & 15 \\
\hline & $\begin{array}{l}\text { Over } 3.5 \text { hours- } \\
\text { under } 7 \text { hours }\end{array}$ & 91 & 19 & & None & 20 & 4 \\
\hline & Over 7 hours- & \multirow{2}{*}{67} & \multirow{2}{*}{13} & & Other & 6 & 1 \\
\hline & under 14 hours & & & \multirow{5}{*}{$\begin{array}{l}\text { Mean personal } \\
\text { monthly income }\end{array}$} & 10,000 NTD or below & 113 & 23 \\
\hline & $\begin{array}{l}\text { More than } 14 \\
\text { hours - under } \\
21 \text { hours }\end{array}$ & 46 & 10 & & 10,001-20,000 NTD & 82 & 17 \\
\hline & $\begin{array}{l}\text { More than } 21 \\
\text { hours }\end{array}$ & 46 & 10 & & $20,001-40,000$ NTD & 200 & 40 \\
\hline \multirow{2}{*}{$\begin{array}{l}\text { Whether one } \\
\text { has online } \\
\text { shopping } \\
\text { experience }\end{array}$} & Yes & 226 & 47 & & 40,001-60,000 NTD & 71 & 15 \\
\hline & No & 258 & 53 & & 60,001 or more & 18 & 4 \\
\hline \multirow{2}{*}{ Marital status } & Married & 274 & 57 & \multirow{2}{*}{ Children } & None & 220 & 45 \\
\hline & Unmarried & 210 & 43 & & Yes & 264 & 55 \\
\hline
\end{tabular}

Most consumers purchased from supermarkets, followed by volume stores, and convenience stores. On average, the most frequently purchased frozen foods were ice-type foods, at $17 \%$, followed by flourbased foods such as dumplings and green onion pancakes (15\%), followed by ball-type foods such as fish balls and meat balls (12\%). The highest purchase frequency was one month (40\%), and the mean purchase amount for most was below 500 NTD (44\%). The three main motives for buying frozen food were purchasing convenience, convenient and quick preparation, and longer preservation (Table 3). 
Table 3. Compilation of buying through low-temperature logistics

\begin{tabular}{|c|c|c|c|c|c|c|c|}
\hline Item & Type & Freq & $\begin{array}{l}\text { Ratio } \\
(\%)\end{array}$ & Item & Type & Freq & Rank \\
\hline \multirow{2}{*}{$\begin{array}{l}\text { Whether low- } \\
\text { temperature } \\
\text { logistics is used }\end{array}$} & Yes & 135 & 28 & \multirow{7}{*}{$\begin{array}{l}\text { Reasons for not using } \\
\text { low-temperature } \\
\text { logistics (multiple) }\end{array}$} & $\begin{array}{l}\text { Cannot see physical } \\
\text { products }\end{array}$ & 1427 & 1 \\
\hline & No & 349 & 72 & & $\begin{array}{l}\text { Concern about poor } \\
\text { quality }\end{array}$ & 1238 & 2 \\
\hline \multirow{7}{*}{$\begin{array}{l}\text { Ratio of purchase } \\
\text { frequency and } \\
\text { amount } \\
\text {--purchase } \\
\text { frequency }\end{array}$} & One month & 192 & 39 & & $\begin{array}{l}\text { Concern about food } \\
\text { storage and hygiene }\end{array}$ & 1055 & 3 \\
\hline & Two weeks & 114 & 23 & & Payment security & 1050 & 4 \\
\hline & Three months & 88 & 19 & & $\begin{array}{l}\text { Does not want to } \\
\text { change purchasing } \\
\text { habits }\end{array}$ & 807 & 5 \\
\hline & One week & 52 & 11 & & $\begin{array}{l}\text { Complex/inconvenie } \\
\text { nt ordering } \\
\text { procedures }\end{array}$ & 637 & 6 \\
\hline & \multirow{2}{*}{$\begin{array}{l}\text { Six months } \\
\text { One year }\end{array}$} & 28 & 6 & & Other & 19 & 7 \\
\hline & & 2 & 0 & \multirow{8}{*}{$\begin{array}{l}\text { Channels for buying } \\
\text { low-temperature foods } \\
\text { (multiple) }\end{array}$} & $\begin{array}{l}\text { Supermarket } \\
\text { Volume }\end{array}$ & 3002 & 1 \\
\hline & Over one year & 8 & 2 & & $\begin{array}{l}\text { stores/wholesale } \\
\text { stores }\end{array}$ & 2885 & 2 \\
\hline \multirow{6}{*}{$\begin{array}{l}\text { Ratio of purchase } \\
\text { frequency and } \\
\text { amount -mean } \\
\text { purchase amount }\end{array}$} & $\begin{array}{l}\text { Less than } 500 \\
\text { NTD }\end{array}$ & 212 & 44 & & Convenience stores & 2442 & 3 \\
\hline & $\begin{array}{l}501- \\
1,000 \text { NTD }\end{array}$ & 179 & 37 & & $\begin{array}{l}\text { Traditional } \\
\text { stores/small stores }\end{array}$ & 728 & 4 \\
\hline & $\begin{array}{l}1,001- \\
2,000 \mathrm{NTD}\end{array}$ & 76 & 16 & & $\begin{array}{l}\text { Military, public } \\
\text { servant, education } \\
\text { benefit centers }\end{array}$ & 713 & 5 \\
\hline & $\begin{array}{l}2,001- \\
3,000 \text { NTD }\end{array}$ & 13 & 3 & & Online shopping & 176 & 6 \\
\hline & \multirow{2}{*}{$\begin{array}{l}\text { More than 3,001 } \\
\text { NTD }\end{array}$} & \multirow{2}{*}{4} & \multirow{2}{*}{0} & & Television shopping & 117 & 7 \\
\hline & & & & & Other & 30 & 8 \\
\hline Item & Type & Freq & Rank & Item & Type & Freq & Rank \\
\hline \multirow{11}{*}{$\begin{array}{l}\text { Types of low- } \\
\text { temperature foods } \\
\text { purchased } \\
\text { (multiple) }\end{array}$} & Ice & 245 & 1 & \multirow{11}{*}{$\begin{array}{l}\text { Ratios of purchase } \\
\text { motive (multiple) }\end{array}$} & Convenient and & 281 & 1 \\
\hline & Flour & 214 & 2 & & fast cooking & & \\
\hline & Fried & 173 & 3 & & Can be preserved & 217 & 2 \\
\hline & Balls & 176 & 4 & & longer & & \\
\hline & Frozen cuisines & 166 & 5 & & Convenient & 324 & 3 \\
\hline & Meats & 149 & 6 & & $\begin{array}{l}\text { purchasing } \\
\text { Many flavors to }\end{array}$ & 148 & 4 \\
\hline & Oden & 104 & 7 & & choose from & & \\
\hline & Rice products & 90 & 8 & & Price is cheap & 100 & 5 \\
\hline & $\begin{array}{l}\text { Frozen fruits } \\
\text { and vegetables }\end{array}$ & 65 & 9 & & Test tastes & 31 & 6 \\
\hline & $\begin{array}{l}\text { Medicinal } \\
\text { cuisines }\end{array}$ & 39 & 10 & & Food nutrition & 5 & 7 \\
\hline & $\begin{array}{l}\text { New Year's } \\
\text { dishes }\end{array}$ & 32 & 11 & & Other & 3 & 8 \\
\hline
\end{tabular}

Note: reasons for not using low-temperature logistics (multiple), channels for buying low-temperature foods (multiple) are ranked $1,2,3 \ldots . .$. , the first place ranking has 3 points, second place has 2 points.......and so on.

\section{Experiences with Low-temperature Logistics Services}

Among the 484 valid questionnaires, 135 indicated experience with low-temperature logistics services. The analysis demonstrated that more participants ordered frozen food with convenience stores as the purchasing channel (46\%), and the mean amount spent was 501-1000 NTD (49\%). The highest purchasing frequency was one month (30\%).
Most selected home delivery as delivery method (70\%), and most selected cash on delivery as payment method (58\%). Twenty-eight percent of participants received the relevant information from television commercials. The top three factors influencing purchases were freshness, expiration date, and service quality. Participants indicated that freshness was the main consideration in using lowtemperature logistics services, followed by ease of goods acquisition, and ease of goods ordering (Table 4). 
Table 4. Low-temperature logistics consumption characteristics

\begin{tabular}{|c|c|c|c|c|c|c|c|}
\hline Item & Tyре & Frequency & Ratio (\%) & Item & Туре & Frequency & $\begin{array}{l}\text { Ratio } \\
(\%)\end{array}$ \\
\hline \multirow{5}{*}{$\begin{array}{l}\text { Purchase } \\
\text { channels }\end{array}$} & $\begin{array}{l}\text { Convenience store } \\
\text { ordering }\end{array}$ & 62 & 46 & \multirow{7}{*}{$\begin{array}{l}\text { Frequency for } \\
\text { buying frozen } \\
\text { food }\end{array}$} & One month & 40 & 29 \\
\hline & \multirow{3}{*}{$\begin{array}{l}\text { Online shopping } \\
\text { Television shopping } \\
\text { Mail order }\end{array}$} & 44 & 32 & & \multirow{5}{*}{$\begin{array}{l}\text { Six months } \\
\text { Three months } \\
\text { More than one } \\
\text { year } \\
\text { Two weeks } \\
\text { One year }\end{array}$} & 35 & 26 \\
\hline & & 17 & 13 & & & 34 & 25 \\
\hline & & 9 & 7 & & & 8 & 6 \\
\hline & Other & 4 & 2 & & & 7 & 5 \\
\hline \multirow{6}{*}{$\begin{array}{l}\text { Mean amount } \\
\text { spent on frozen } \\
\text { food }\end{array}$} & $\begin{array}{l}501- \\
1,000 \text { NTD }\end{array}$ & 67 & 49 & & & 6 & 4 \\
\hline & $\begin{array}{l}1,001- \\
2,000 \text { NTD }\end{array}$ & 33 & 24 & & One week & 6 & 4 \\
\hline & Under 500 NTD & 30 & 22 & \multirow{4}{*}{$\begin{array}{l}\text { Delivery } \\
\text { methods for } \\
\text { purchased } \\
\text { frozen food }\end{array}$} & \multirow{2}{*}{$\begin{array}{l}\text { Home delivery } \\
\text { Pick-up at store }\end{array}$} & 94 & 69 \\
\hline & & 4 & 3 & & & 40 & 29 \\
\hline & More than 3,001 & & & & Other & & \\
\hline & N1D & 2 & 2 & & & 2 & 1 \\
\hline \multirow{5}{*}{$\begin{array}{l}\text { Payment } \\
\text { methods for } \\
\text { purchased frozen } \\
\text { food }\end{array}$} & Cash on delivery & 79 & 58 & \multirow{5}{*}{$\begin{array}{l}\text { Sources of } \\
\text { finding out } \\
\text { about frozen } \\
\text { food }\end{array}$} & $\begin{array}{l}\text { Television } \\
\text { commercial }\end{array}$ & 67 & 28 \\
\hline & Credit card & 25 & 18 & & $\begin{array}{l}\text { Heard from } \\
\text { others }\end{array}$ & 64 & 27 \\
\hline & ATM transfer & 19 & 14 & & $\begin{array}{l}\text { Webpage } \\
\text { recommend- } \\
\text { dation }\end{array}$ & 60 & 25 \\
\hline & Postal transfer & 11 & 8 & & $\begin{array}{l}\text { Newspapers and } \\
\text { magazines }\end{array}$ & 42 & 17 \\
\hline & Other & 2 & 2 & & $\begin{array}{l}\text { Company } \\
\text { website }\end{array}$ & 8 & 3 \\
\hline \multirow[t]{4}{*}{ Item } & Type & Frequency & Ranking & \multirow{12}{*}{$\begin{array}{l}\text { low- } \\
\text { temperatu } \\
\text { foods }\end{array}$} & Type & Frequency & Ranking \\
\hline & Freshness & 523 & 1 & & Freshness & 742 & 1 \\
\hline & $\begin{array}{l}\text { Ease in acquiring } \\
\text { goods }\end{array}$ & 495 & 2 & & Expiration date & 610 & 2 \\
\hline & $\begin{array}{l}\text { Ease in ordering } \\
\text { goods }\end{array}$ & 382 & 3 & & Service quality & 415 & 3 \\
\hline \multirow{8}{*}{$\begin{array}{l}\text { Reasons for using } \\
\text { low-temperature } \\
\text { logistics to } \\
\text { purchase frozen } \\
\text { food }\end{array}$} & Expiration date & 314 & 4 & & $\begin{array}{l}\text { Ease in acquiring } \\
\text { goods }\end{array}$ & 331 & 4 \\
\hline & Payment choice & 288 & 5 & & $\begin{array}{l}\text { Ease in ordering } \\
\text { goods }\end{array}$ & 313 & 5 \\
\hline & Designated time & 282 & 6 & & Price & 283 & 6 \\
\hline & Service quality & 272 & 7 & & Payment choice & 228 & 7 \\
\hline & Price & 254 & 8 & & Time-efficiency & 121 & 8 \\
\hline & Time-efficiency & 176 & 9 & & Designated time & 81 & 9 \\
\hline & Evening delivery & 129 & 10 & & Delivery tracking & 65 & 10 \\
\hline & Delivery tracking & 109 & 11 & & Evening delivery & 40 & 11 \\
\hline
\end{tabular}

\section{Chi-square Test}

To understand whether subject characteristics affect frozen food purchases, a crossover analysis and chisquare test were performed to determine the consumers' purchase characteristics and personal traits. Table 5 demonstrates that among the 60 groups of chi-square tests, 23 groups showed significant differences.

The analytical results of the chi-square tests were used to formulate the study's basic hypotheses, as follows:

H1: Participants with different characteristics used low-temperature logistics services to buy frozen food at different frequencies.
Evaluation results: partially established.

Explanation: Only significant variance in age, education, and time spent online existed, the other variables did not demonstrate significant variance. Consumers 20-29 years old had a high purchasing frequency, and participants with high (vocational) school education levels had a higher purchasing frequency. Participants who spent less than 3.5 hours online demonstrated higher purchasing frequency.

$\mathrm{H}_{2}$ : Participants with different characteristics differ in the mean purchase amount spent buying frozen food from low-temperature logistics companies.

Evaluation results: partially established.

Explanation: Only occupation and monthly personal 
Table 5. Chi-square test chart

\begin{tabular}{|c|c|c|c|c|c|c|}
\hline \multirow[b]{2}{*}{ Variable } & \multirow[b]{2}{*}{$\begin{array}{l}\text { Purchase } \\
\text { frequency }\end{array}$} & \multirow{2}{*}{$\begin{array}{l}\text { Mean } \\
\text { purchase } \\
\text { amount }\end{array}$} & \multirow{2}{*}{$\begin{array}{l}\text { Whether one has } \\
\text { experience using low- } \\
\text { temperature } \\
\text { logistics }\end{array}$} & \multicolumn{3}{|c|}{ Experience using low-temperature logistics } \\
\hline & & & & $\begin{array}{l}\text { Which type of } \\
\text { channel used }\end{array}$ & $\begin{array}{c}\text { How goods are } \\
\text { acquired }\end{array}$ & $\begin{array}{l}\text { Payment } \\
\text { methods }\end{array}$ \\
\hline Gender & 0.131 & 0.585 & $0.003^{*}$ & 0.853 & 0.462 & 0.474 \\
\hline Age & $0.042^{*}$ & 0.171 & $0.000^{*}$ & $0.009^{*}$ & 0.255 & $0.001^{*}$ \\
\hline Residence & 0.447 & 0.197 & $0.030^{*}$ & 0.172 & 0.795 & 0.312 \\
\hline Education & $0.007^{*}$ & 0.729 & $0.000^{*}$ & 0.279 & $0.001^{*}$ & 0.244 \\
\hline Occupation & 0.138 & $0.000^{*}$ & $0.015^{*}$ & 0.201 & 0.631 & 0.909 \\
\hline Marital status & 0.437 & 0.128 & $0.033^{*}$ & 0.064 & 0.686 & 0.073 \\
\hline $\begin{array}{l}\text { Whether there are } \\
\text { children }\end{array}$ & 0.541 & 0.088 & $0.018^{*}$ & 0.060 & 0.848 & 0.075 \\
\hline $\begin{array}{l}\text { Personal monthly } \\
\text { income }\end{array}$ & 0.302 & $0.003^{*}$ & $0.000^{*}$ & 0.716 & 0.200 & 0.131 \\
\hline Time spent online & $0.004^{*}$ & 0.068 & $0.000^{*}$ & 0.074 & $0.032^{*}$ & $0.006^{*}$ \\
\hline Online shopping & 0.109 & 0.856 & $0.000^{*}$ & $0.001^{*}$ & $0.002^{*}$ & $0.008^{*}$ \\
\hline
\end{tabular}

Note: * denotes significant difference

Table 6. Binary logistic regression model coefficient calibration results

\begin{tabular}{lcccccc}
\hline & $\beta$ & S.E. & Wald & $\begin{array}{c}\text { Degrees of } \\
\text { freedom }\end{array}$ & Significance & $\operatorname{Exp}(\beta)$ \\
\hline 40-49 years of age & -0.761 & 0.301 & 6.402 & 1 & 0.011 & 0.467 \\
New Taipei City (urban area) & -0.976 & 0.256 & 14.544 & 1 & 0.000 & 0.377 \\
high (vocational) school & -1.024 & 0.275 & 13.878 & 1 & 0.000 & 0.359 \\
junior college and university & -1.815 & 0.306 & 35.069 & 1 & 0.000 & 0.163 \\
10,000 and under & -2.172 & 0.365 & 35.456 & 1 & 0.000 & 0.114 \\
does not use the internet & -0.958 & 0.302 & 10.028 & 1 & 0.002 & 0.384 \\
has online shopping experience & 2.337 & 0.300 & 60.562 & 1 & 0.000 & 10.350 \\
\hline
\end{tabular}

income demonstrated significant differences, the other variables did not show significant differences. Among participants with commerce/service occupations, the mean purchase amount was higher. Participants with monthly personal income of 20,00140,000 NTD had higher mean purchase amounts.

$\mathrm{H}_{3}$ : Participants with different characteristics differ in frozen food purchase experiences with lowtemperature logistics companies.

Evaluation results: fully established.

Explanation: The chi-square test results indicated significant trait differences. More customers were women, and most people in the 20-29 age group had online purchasing experience. Most were residents of the New Taipei City, and most participants with high (vocational) school education levels had online purchasing experience. Participants with commerce/ service occupations had more purchasing experience, as well as married participants and participants with children. Participants with monthly personal income of 20,001-40,000 NTD had online purchasing experience, and most spent less than 3.5 hours a week online.

$\mathrm{H}_{4}$ : Participants with different characteristics used different purchasing channels to buy frozen food from low-temperature logistics companies.
Evaluation results: partially established.

Explanation: The test results only demonstrated significant age differences in an online shopping experience, and no significant differences were evident in the other variables. Participants in the 20-29 age group used online shopping more frequently to order frozen food, and participants in the 30-39 age group ordered through convenience stores more frequently. $\mathrm{H}_{5}$ : Participants with different characteristics selected different delivery methods for their frozen food purchases from low-temperature logistics companies.

Evaluation results: partially established.

Explanation: The test results only revealed significant differences in education, time spent online, and online shopping experience, and no significant differrences existed in the other variables. Participants with elementary school or lower educational levels more frequently used to store deliveries, and participants with elementary school and higher educational levels tended to use home delivery. Participants who did not use the internet or spent more than 21 hours a week online used store deliveries more regularly. More than $70 \%$ of participants with online shopping experience used home delivery, and participants without online shopping experience were split between home delivery and store collection. 
$\mathrm{H}_{6}$ : Participants with different characteristics used different payment methods to buy frozen food from low-temperature logistics companies.

Evaluation results: partially established.

Explanation: The test results demonstrated three significant differences (age, time spent online, and online shopping) and the other variables demonstrated no significant differences. More than half of the participants used cash on delivery as payment method. However, participants in the 15-19 age groups who used the internet for 3.5-7 hours a week were more likely to use the cash on delivery payment method than participants who used the internet for 14-21 hours a week, reaching 70\%. Other payment methods used were credit cards and ATMs, and participants without online shopping experience primarily paid by postal transfer.

\section{Logistic Regression}

To analyze the crucial factors that influence consumers use low-temperature logistics companies to purchase frozen food, this study used a binary logistic regression model to establish a predictive model for usage intentions.

\section{Basic Variables}

After integrating the questionnaire results, frozen food purchasing experience using low-temperature logistics companies was used as the dependent variable, and the socioeconomic and internet usage traits of participants as independent variables. The independent variables included the following: (1) The socioeconomic traits of the participants were gender, age, occupation, place of residence, education, occupation, marital status, number of children, and monthly income. (2) The Internet usage traits of the participants were time spent online each week, and whether a subject had online shopping experience.

\section{Results of Model Construction}

We constructed a binary logistic regression model using forward stepwise regression, and the coefficient calibration results are demonstrated in Table 6. Table 6 indicates that the main variables that affect low-temperature logistics companies included age, place of residence, education, monthly income, whether participants had online shopping experience, and time spent online each week.

\section{Influence of Personal Socioeconomic Conditions}

Participants aged 40-49 had the lower purchasing intention than participants over 60 years old. Participants aged 40-49 years old were 0.467 times more likely to use low-temperature logistics companies to
Table 7. Nagelkerke $R$ square values

\begin{tabular}{ccc}
\hline$-2 \log$ likelihood & Cox \& Snell R square & Nagelkerke R square \\
\hline 435.859 & 0.385 & 0.513 \\
\hline
\end{tabular}

Table 8. Model prediction accuracy rate

\begin{tabular}{|c|c|c|c|c|}
\hline \multirow{3}{*}{ Variable } & & \multicolumn{3}{|c|}{ Prediction } \\
\hline & & \multicolumn{2}{|c|}{$\begin{array}{l}\text { Whether one has the } \\
\text { experience of using } \\
\text { low-temperature } \\
\text { logistics }\end{array}$} & \multirow[t]{2}{*}{$\begin{array}{l}\text { Percentage } \\
\text { modification }\end{array}$} \\
\hline & & No & Yes & \\
\hline $\begin{array}{l}\text { Whether one has the } \\
\text { experience of using }\end{array}$ & No & 304 & 45 & 87.1 \\
\hline $\begin{array}{l}\text { low-temperature } \\
\text { logistics }\end{array}$ & Yes & 51 & 84 & 62.2 \\
\hline Percentage & & & 80.2 & \\
\hline
\end{tabular}

buy frozen food than participants who were over 60 . Participants who were residents of the New Taipei City metropolitan area had lower purchase intention than participants residing in the suburbs of the New Taipei City. Residents of New Taipei City metropolitan areas were 0.377 times more likely than participants living in the suburbs to buy frozen food from low-temperature logistics companies. Participants with high (vocational) school, junior college, and university educational levels had lower purchasing intention than participants who attended graduate school and higher. Participants with education levels of high (vocational) school and junior college and university were 0.359 and 0.163 times more likely than participants who attended graduate school and over to buy frozen food from low-temperature logistics companies. Participants with monthly income levels below 10,000 NTD had lower purchasing intention than participants with monthly income levels of 60,000 NTD and above. Participants with monthly income levels of less than 10,000 NTD were 0.114 times more likely to use buy frozen food from low-temperature logistics companies than participants with monthly income levels of 60,000 NTD and more.

\section{Influence of Internet Usage Traits}

Participants with online shopping experience had greater purchasing intention than those without. Participants with online shopping experience were 10.350 times more likely than those without to buy frozen food from low-temperature logistics companies. The "time spent online" results indicated that fewer participants did not use the Internet at all compared to participants who used it longer than 21 hours a week. Participants who did not use the Internet were 0.384 times more likely to buy frozen food from lowtemperature logistics companies than participants who spent more than 21 hours online.

The Nagelkerke R2 (Table 7) value in the regression model was 0.513 , which indicated that the model 
results were acceptable, but not outstanding. However, the predictive accuracy of this model reached $80.2 \%$, which indicated that this model has considerable predictive ability (Table 8).

\section{Conclusions}

The purpose of this study was to explore the characteristics of consumers who used online shopping to purchase frozen food products, and stratified sampling and interview questionnaires were used to collect data. Participants were 15 years and older and were residents of Taipei City and New Taipei City. Five hundred and eighty four questionnaires were distributed, and 484 valid questionnaires were returned, demonstrating a valid questionnaire retrieval rate of $88.32 \%$.

The analytical results indicated that purchase motive, types of products purchased, purchase frequency, purchase experience, purchase channels, delivery methods, and payment methods all partially influenced how consumers buy frozen foods through low-temperature logistics companies. Most consumers that shopped online for frozen products worked in the commerce or service industries and were in the 20 to 49 age group, and a greater proportion of participants were women.

One-third of the participants had experience buying frozen foods through low-temperature logistics companies. These purchases were primarily influenced by the freshness, ease of acquiring goods, and ease of ordering goods considerations.

The scope of this research was limited to Taipei City and New Taipei City, and future research can focus on online consumer characteristics in different geographic locations, consumer characteristics in different industries, and whether consumer characteristics vary by location.

The results of this study demonstrated that twothirds of participants currently do not use online shopping to buy frozen food, and the main reasons were because they could not see the actual product, and they had food quality, storage, hygiene, and transaction security concerns. Therefore, it is suggested that companies selling frozen products online should focus on how to provide consumers with channels to experience the physical products, provide quality assurances, and implement more secure transaction methods.

Participants who purchased frozen food through lowtemperature logistics companies placed the greatest importance on freshness. Therefore, temperature control was critical to delivery logistics. It is sug- gested that logistics and delivery businesses should improve temperature controls, and online shopping vendors should also select logistics and delivery businesses that adequately control product temperatures to maintain optimal product quality.

\section{References}

1. Aaker, D.A., Strategic Market Management, New York: John Wiley and Sons Inc., pp.27-35, 201276, 1988.

2. Chang, Y.M., Effect of Market Orientation on New Product Performance Cases study of Freezing Industry in Taiwan, Department of Business Administration, Chung Yuan Christian University, R. O. C., Master paper, 2004.

3. Chen, H.I., A Study of the Strategic Groups in Frozen Prepared Food Industry and their Performance, Department of Food Science, Tunghai University, R. O. C., Master Paper, 2005.

4. Department of Statistics, Ministry of Economic Affairs, Yearbook of Industrial Production in Taiwan, pp.107-108, 2012.

5. Guo, S.Y., A Study on Relationship between Consumer Characteristics and Internet Shopping Intention: Using Pershiable Food Products as an Example, Department of Marketing Management, National Chung Hsing Universisty, R. O. C., Master Paper, 2001.

6. Hu, K.C., Wu, Hsiao-Yu, The Effects of Brand Alliance Fitness on Brand Image and Customer's Purchase Intentions for Home-Delivery Industry, Web Journal of Chinese Management Review, 12(2), 2009, pp.1-21.

7. Kuo, J.C, and Chung, J.C, Cold Low-Temperature Home Delivery System, Chinese Water, Electrical, Frozen and Air Conditioning, (1), 2006, pp.1-3,

8. Lan, J.P., A Study of Productive and Marketing strategy of Home-delivery, Department of Civil Engineering, National Central University, R. O. C., Master Paper, 2002.

9. Lee, J.J, Customer Satisfaction and the Dimensions of Service Quality -An Empirical Study of Home-Delivery Industry, Department of Business Administration, National Taipei University, R. O. C., Master Paper, 2004.

10. Lin, C. F., Analysis of Consumer Use of Both the Virtual Channel and Physical Channel Adopted by Convenience Stores, Executive Master of Business Administration, Providence University, R. O. C., Master Paper, 2006.

11. Lu, H.C., The Study on Business Strategy of Professional Home Delivery Carriers, Department Business and Operations Managment, Graduate School, R. O. C., Master Paper, 2003.

12. Shiaw, M.S., and Chiang, H.L., The Impacts of Characteristics of Consumers, E-Business, Pro- 
duct, and the Internet Environment on Online Shopping Behavior, Journal of Information Management-Concepts, Systems, and Applications, 7(2), 2005, pp. 71-90.

13. Wang, C. M., Developmental Trends in Frozen Food, Food Marketing, 8702, 1998, pp.3.

14. Wang, P.Y., Modeling and Solving the Frozen Food Distribution Problem, Department of Industrial Engineering and Management, Chaoyang University of Technology, R. O. C., Master Paper, 2000.
15. Yeh, Y. L., 2005, The Purchasing Effects of Customer Behavior on Customer Satisfaction and Customer Loyalty through an Empirical Study of Frozen Prepared Foods, Department of International Business, Chung Yuan Christian University, R. O. C., Master Paper, 2005.

16. Yu, J.C., The Study of the Key Factors to Operate Door-to-door Business, Graduate Institute of Management, National Taiwan University of Science and Technology, R. O. C., Master Paper, 2006. 